\title{
Application of sonication- and vacuum infiltration- assisted Agrobacterium-mediated transformation of rice embryo (Oryza sativa L.)
}

\author{
Touchkanin Jongjitvimol ${ }^{\mathrm{a}}$, Kawee Sujipuli ${ }^{\mathrm{b}}$, Sittichai Urtgam ${ }^{\mathrm{a}, *}$ \\ a Biology Program, Faculty of Science and Technology, Pibulsongkram Rajabhat University, \\ Phitsanulok 65000 Thailand \\ b Department of Agricultural Science, Faculty of Agriculture, Natural Resources and Environment, \\ Naresuan University, Phitsanulok 65000 Thailand
}

*Corresponding author, e-mail: sittichai.u@psru.ac.th

Received 5 Dec 2019

Accepted 6 Jun 2020

\begin{abstract}
Rice transgenics could be performed based on Agrobacterium-mediated transformation which could improve the rice breeding programs to settle for diseases, insect pests and the climate changes. In this study, the new strategy of combination of sonication-assisted Agrobacterium-mediated transformation (SAAT) and vacuum infiltrationassisted Agrobacterium-mediated transformation (VIAAT) system for producing transgenic rice was developed. This research demonstrated an Agrobacterium-mediated transformation protocol for whole rice seeds by employing both sonication and vacuum infiltration. Two Agrobacterium tumefaciens strains AGL1 and EHA105, carrying a binary vector pCAMBIA1301 (with a gusA-intron) or pCAMBIA1304 (with a gusA), were used to examine for embryo transformation efficiency of the three rice grain cultivars, RD41, RD47 and PSL2. The results show that (1) SAAT for 10 min was proved to be the best for increasing microwound regions on the embryo surface; (2) EHA105-pCAMBIA1304 gave higher transformation efficiency of dehusked seeds (87.5 $\pm 7.5 \%)$ than AGL1-pCAMBIA1304 (37.5 $\pm 2.5 \%)$; $(3)$ the combination of SAAT for $10 \mathrm{~min}$ followed by another $10 \mathrm{~min}$ of VIAAT gave the transient GUS expression in coleoptile (18\%) and primary leaf (2\%) of husked seedling; and (4) the use of transformation protocol with EHA105pCAMBIA1301 showed that the rice cultivar PSL2 gave significantly higher transformation efficiency than RD41 and RD47. This work thus suggests that genetic transformation in plants could be achieved by SAAT and VIAAT techniques without tissue culture process.
\end{abstract}

KEYWORDS: Oryza sativa, Agrobacterium, sonication-assisted transformation, vacuum infiltration-assisted transformation, gusA

\section{INTRODUCTION}

Rice (Oryza sativa L.) is one of the most economically important cereal crops of Thailand, but the main reduction of rice (O. sativa L.) quality and quantity has been greatly affected by diseases, insect pests and the climate changes. Many conventional breeding programs have made a significant contribution to create and improve desirable agronomic traits in rice; however, these require tremendous, labor-intensive and time-consuming process. To solve these problems, the development of modern biotechnology techniques such as Agrobacterium-mediated transformation technology has made the generation of transgenic rice simpler and more reliable [1]. This is because Agrobacterium-mediated transformation provides a high op- portunity to transfer large DNA fragments, carrying genes without any rearrangement, which is very useful to study their cumulative and interactive effects on polygenic traits. Moreover, it has high inserted transgene as a single or low copy into plant genome [2], which may give high efficiency for gene expression in the next generation [3, 4]. Mostly, Agrobacterium-mediated transformation protocols for plants are based on tissue culture techniques, which usually have some limitation such as long process, skilled labor and expensive laboratory equipment [5]. In contrast, in planta transformation such as sonication- and vacuum infiltration-assisted Agrobacterium-mediated transformation (SAAT and VIAAT) can directly transfer gene into a plant with no requirement of tissue culture techniques [6]. The SAAT and VIAAT methods have been successfully 
employed in enhancing transformation rates in banana cv. Rasthali [7], Vigna unguiculata [6], Lens culinaris [8] and Indian soybean [9].

In this study, for the first time, we report the development of the use of the combination of SAAT and VIAAT systems in rice embryo, so that this protocol can be potentially applied in routine experiments for further producing transgenic rice.

\section{MATERIALS AND METHODS}

\section{Chemicals}

Rifampicin, kanamycin, 6-benzylaminopurine (BAP), MS medium and 5-bromo-4-chloro-3indolyl- $\beta$-D glucuronidase (X-gluc) were purchased from Sigma, USA. Taq DNA polymerase was from Vivantis, Malaysia.

\section{Rice sample preparation}

Three rice cultivars (standard paddy rice RD41, RD47 and PSL2) used in this study were obtained from Phitsanulok Rice Research Center, Thailand. Mature rice seeds at least $2 \mathrm{~kg}$ were dehusked by rice-milling machine model NW 1000 TURBO (Natawee Technology, Thailand), and only complete seeds (with embryo) were selected to use for transformation experiments whereas husked seeds were used as a control group.

\section{Binary plasmid, Agrobacterium strain and culture condition}

Two strains of Agrobacterium tumefaciens, AGL1 and EHA105 harboring pCAMBIA1301 or pCAMBIA1304 (kindly provided by Prof. Sompong Techato), were used for rice transformation experiments. Both Agrobacteria contained rifampicin and chloramphenicol resistant genes in bacterial chromosome. The T-DNA region of both binary vectors contains a marker gene (hygromycin phosphotransferase II; $h p t I I$ ) and a reporter gene (betaglucuronidase; gusA in pCAMBIA1304 or gusAintron in pCAMBIA1301). All these genes were driven by the cauliflower mosaic virus (CaMV) $35 \mathrm{~S}$ promoter (Fig. 1). The Agrobacterium was maintained on LB medium ( $1 \%(\mathrm{w} / \mathrm{v})$ tryptone, $0.5 \%$ $(\mathrm{w} / \mathrm{v})$ yeast extract and $1 \%(\mathrm{w} / \mathrm{v}) \mathrm{NaCl})$ supplemented with $40 \mu \mathrm{g} / \mathrm{ml}$ rifampicin and $50 \mu \mathrm{g} / \mathrm{ml}$ kanamycin, and then incubated at $28^{\circ} \mathrm{C}$ for $48 \mathrm{~h}$. A single bacterial colony was cultured into $25 \mathrm{ml}$ of liquid LB medium supplemented with $25 \mu \mathrm{g} / \mathrm{ml}$ rifampicin and $50 \mu \mathrm{g} / \mathrm{ml}$ kanamycin and incubated at $28-30^{\circ} \mathrm{C}$ for $24-48 \mathrm{~h}$ with shaking at $200 \mathrm{rpm}$
(Shaking Incubator model SI-23MC; Bioer Technology, China). The culture was measured at $600 \mathrm{~nm}$ $\left(\mathrm{OD}_{600}\right)$ until it reached 0.8 . The cell pellets were harvested by centrifugation at $3512 \times \mathrm{g}$ for $3 \mathrm{~min}$ at $25^{\circ} \mathrm{C}$ and resuspended in a final volume of $100 \mathrm{ml}$ standard inoculation medium (SIM), modified from Clough et al [10] ( $1 \frac{1}{2} \mathrm{MS}$ medium, 5\% sucrose, $44 \mathrm{nM}$ 6-benzylaminopurine (BAP) and $0.075 \%$ Tween-20, adjusted $\mathrm{pH}$ to 5.7).

\section{Primer sets of gusA-intron and gusA detection using PCR}

Three primer sets used to amplify the gusAintron and gusA region were designed from entire sequences of pCAMBIA-1301 (AF234297.1) and pCAMBIA-1304 (AF234300.1), respectively, which are available on NCBI website. The primer set no. 1 was gusA-F1 (5'-CAACGAACTGAACTGGCAGA-3') and gusA-R1 ( $5^{\prime}$-TCTCTTTGATGTGCTGTGCC- $3^{\prime}$ ), giving PCR product of 989 bp length in both PCAMBIA-1301 and pCAMBIA-1304, primer set no. 2 was gusA-F2 ( $5^{\prime}$-TGCGTCACAGCCAAAAGC- $3^{\prime}$ ) and gusA-R2 (5'-CTCGCATTACCCTTACGC-3'), giving PCR product of 245 bp length only in pCAMBIA1304 and primer set no. 3 was gusA-F1 and gusAR2 giving PCR product of 597 bp length only in pCAMBIA-1301.

\section{PCR condition}

Genomic DNA (gDNA) was extracted from a single colony of each Agrobacterium strain, and the PCR reaction was performed in a total volume of $20 \mu \mathrm{l}$ containing $100 \mathrm{ng}$ gDNA, $200 \mu \mathrm{M}$ dNTP mix, 1X ViBuffer A, $1.5 \mathrm{mM} \mathrm{MgCl}_{2}$, 1 unit Taq DNA polymerase and $200 \mathrm{mM}$ of each specific primer. The PCR was carried out in T100 ${ }^{\mathrm{TM}}$ Thermal Cycler (BioRad, USA) with optimal condition profile as predenaturation at $95^{\circ} \mathrm{C}$ for $3 \mathrm{~min}$, amplified by 35 cycles of denaturing at $95^{\circ} \mathrm{C}$ for $30 \mathrm{~s}$, annealing at $55^{\circ} \mathrm{C}$ for $30 \mathrm{~s}$ and extension at $72{ }^{\circ} \mathrm{C}$ for $60 \mathrm{~s}$, and followed by final extension at $72^{\circ} \mathrm{C}$ for $5 \mathrm{~min}$. The PCR products were electrophoresed on $1 \%(\mathrm{w} / \mathrm{v})$ agarose gel and visualized by $0.001 \%$ ethidium bromide staining.

\section{Transformation using SAAT and VIAAT treatments}

The rice seeds were soaked in water overnight. After air drying for an hour, the rice samples were immersed in SIM individually containing Agrobacterium strain EHA105-pCAMBIA1301, AGL1-pCAMBIA1301, EHA105-pCAMBIA1304 or 

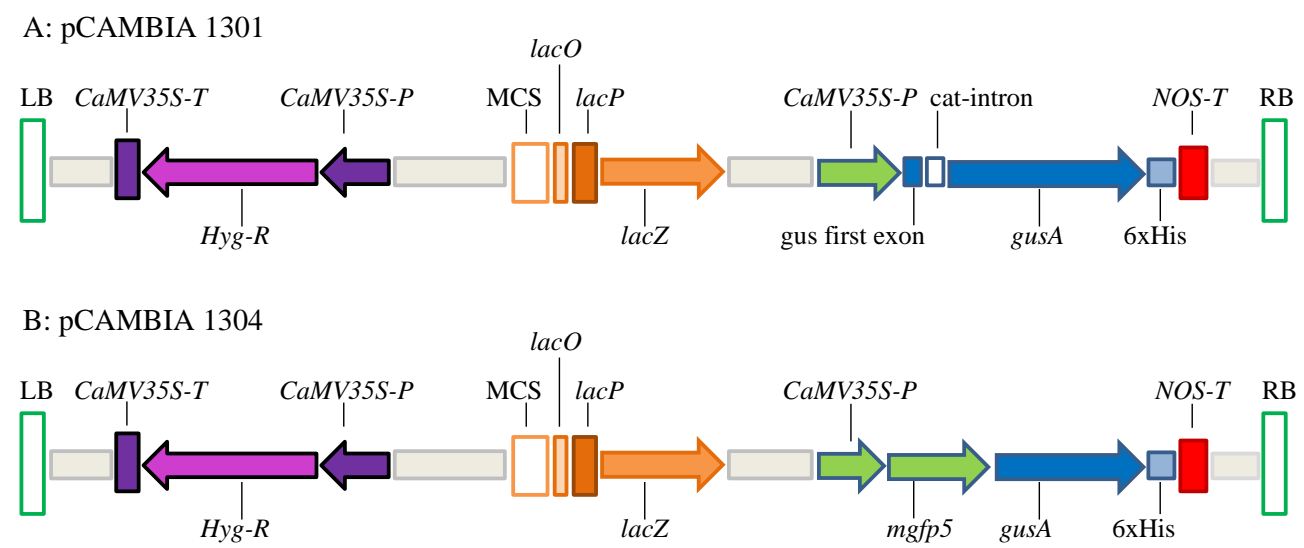

Fig. 1 T-DNA mapping in (A) pCAMBIA1301 [11] and (B) pCAMBIA1304 [12] binary vectors. Arrow indicates the direction of RNA transcription; $\mathrm{RB}=$ right border; $\mathrm{LB}=$ left border; gusA = beta-glucuronidase reporter gene; CaMV35S- $P=$ Cauliflower mosaic virus 35S RNA gene promoter; cat-intron = catalase intron received from castor bean catalase gene; NOS- $T=$ Nopaline synthase terminator $(0.25 \mathrm{~kb}) ; H y g-R=$ Hygromycin B phosphotransferase gene; CaMV35S- $T=$ poly A terminator $(0.3 \mathrm{~kb}), m g f p 5=$ modified green fluorescence protein reporter gene; and MCS $=$ the multiple cloning sites pUC18 polylinker.

AGL1-pCAMBIA1304. They were placed in a bathtype sonicator model WUC-D22H (Daihan Scientific, Korea) and then subjected to ultrasound at a frequency of $30 \mathrm{kHz}$ for $5-10 \mathrm{~min}$. After that, the rice samples were placed in a vacuum oven model VWR 1410 (Sheldon Manufacturing, Inc., USA) consisting of a vacuum pump at 27 inch of mercury for 5-10 min. At the end of treatments, explants were removed from the tubes, placed on sterile filter paper surface to blot off excess bacteria and then transferred to co-cultivation in SIM for 1 day [13].

\section{Histochemical GUS assays}

Transient gusA (or gusA-intron) expression was examined using GUS activity according to histochemical assay [14]. The seed samples were immersed in $90 \%$ acetone for $1 \mathrm{~h}$ and removed to incubate at $37^{\circ} \mathrm{C}$ overnight in GUS staining solution containing $50 \mathrm{mg} / \mathrm{ml}$ 5-bromo-4-chloro-3-indolyl- $\beta$-D glucuronidase (X-gluc), 10 mM EDTA (pH 7), $0.1 \mathrm{M}$ $\mathrm{Na}_{2} \mathrm{PO}_{4}, 0.5 \mathrm{mM} \mathrm{K}_{4}\left(\mathrm{~F}_{4}(\mathrm{CN})_{6}\right), 10 \%$ Triton X-100 and $20 \%$ methanol. After removal of GUS solution, the stained seeds were washed once with mixed solution of methanol:acetic acid (3:1) at room temperature $\left(25-28^{\circ} \mathrm{C}\right)$ overnight. The samples were transferred into $70 \%$ ethanol and stored at $4{ }^{\circ} \mathrm{C}$ at least $2 \mathrm{~h}$. The transient GUS activity of seeds was determined as blue spots under the stereo microscope model Olympus S2H10 with camera model
Olympus SZX7 (Olympus, Japan). The transformation efficiency (\%) was calculated according to the equation;

$$
\text { Efficiency }(\%)=\frac{\text { No. of GUS-stained seeds }}{\text { Total no. of seeds }} \times 100 \text {. }
$$

\section{RESULTS AND DISCUSSION}

To develop the gene transformation method in rice seed, two strains of Agrobacterium (AGL1 and EHA105) carrying individual pCAMBIA1301 or pCAMBIA1304 were used to examine transformation efficiency in rice embryo by using SAAT followed by VIAAT treatments.

\section{Confirmation of gusA-intron and gusA in T-DNA region of binary vectors}

The gusA-intron in pCAMBIA1301 and gusA in pCAMBIA1304 of Agrobacterium strains AGL1 and EHA105 were detected by using colony PCR with three sets of specific primers (as given above). The results showed that primer set no. 1 gave an expected PCR-product size of approximately 989 bp length in both binary vectors (Fig. 2; lanes 2-5). For primer set no. 2, the amplified fragment gave an expected band at approximately 245 bp length in only pCAMBIA-1304 but not in pCAMBIA-1301 (Fig. 2; lanes 6-7). For primer set no.3, the amplified fragment gave an expected band at approximately 597 bp length in only pCAMBIA-1301 but not in pCAMBIA-1304 (Fig. 2; lanes 8-9). These results 


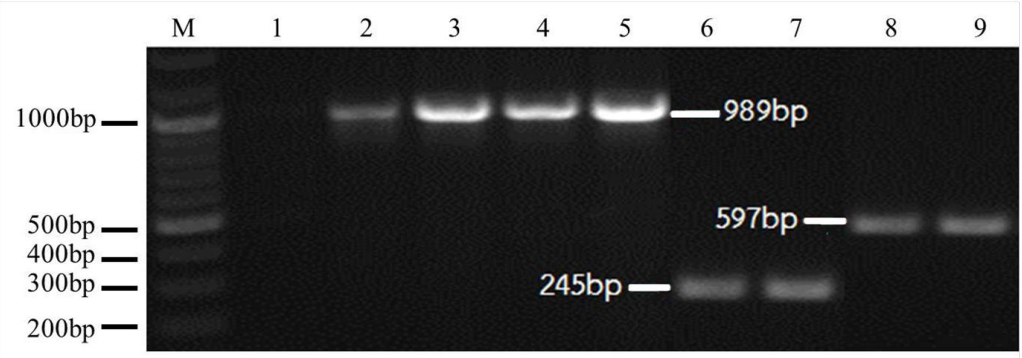

Fig. 2 The confirmation of the presence of gusA-intron and gusA gene in pCAMBIA1301 and pCAMBIA1304, respectively, using colony PCR with 3 primer sets. Lane M represents 100 bp DNA ladder. The Agrobacterium strains AGL1pCAMBIA1301 (lanes 2 and 8), AGL1-pCAMBIA1304 (lanes 3 and 6), EHA105-pCAMBIA1301 (lanes 4 and 9), and EHA105-pCAMBIA1304 (lanes 5 and 7). PCR products were separated on a 1\% agarose gel, and DNA bands were visualized under UV light. The PCR fragments of approximately 989 bp (lanes 2-5), 245 bp (lanes 6-7) and 597 bp (lanes 8-9) were observed using primer pair F1-R1, F2-R2 and F1-R2, respectively. Lane 1 represents a negative control.
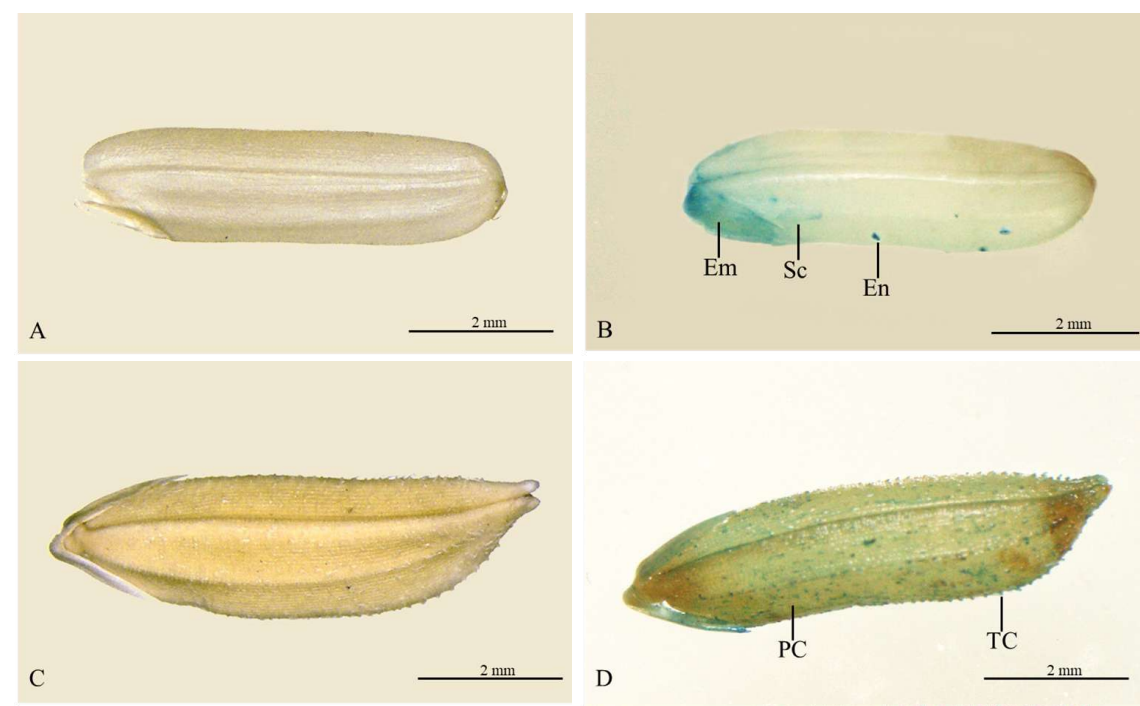

Fig. 3 Histochemical assay of GUS transient expression after gene transformation of gusA gene via SAAT, followed by VIAAT (A) a control group of dehusked seed, (B) GUS-stained in embryo (Em), endosperm (En) and seed coat (Sc) of dehusked seed, (C) a control group of husked seed and (D) GUS-stained trichome (TC) and pericarp (PC) of husked seed.

indicated that pCAMBI-1301 and pCAMBIA-1304 contained gusA-intron and gusA gene, respectively. The gusA-intron can be expressed only in eukaryotic cells but not in prokaryotic cells including A. tumefaciens cells. Therefore, they can be used to monitor efficiency of gene transformation.

\section{Effect of husked and dehusked mature seeds of rice on gene transformation efficiency}

The dehusked or husked seeds were immersed in Agrobacterium strain AGL1-pCAMBIA1304 and subjected to SAAT for $10 \mathrm{~min}$ and followed by VIAAT for $10 \mathrm{~min}$. The result showed that the gusA gene was expressed and located in seed coat $(97 \pm 1 \%)$, endosperm $(6 \pm 2 \%)$ and embryo $(4 \pm 2 \%)$ of dehusked seeds, but expressed only in pericarp (100\%) and trichome (100\%) of husked seeds (Fig. 3). This indicated that the pericarp can be resistant to Agrobacterium infection into the endosperm and embryo of husked seeds because the pericarp has a hard structure consisting of high cellulose, hemicellulose and lignin contents [15]. Moreover, this result may indicate that Agrobacterium can infect the dehusked seeds which causes microwounds within and on the tissue [16], inducing phenolic-compound synthesis [17] and vacuum infiltration might allow better access and infection of plant cells by Agrobacterium [8]. 
Table 1 Influence of dehusked and husked seeds on transformation efficiency using Agrobacterium-mediated transformation via SAAT and VIAAT combination.

\begin{tabular}{lcc}
\hline \multirow{2}{*}{ Agrobacterium strain } & \multicolumn{2}{c}{ Transformation efficiency (\%) } \\
\cline { 2 - 3 } & Control & SAAT \& VIAAT \\
\hline AGL1-pCAMBIA1304 & $17.0 \pm 2.0^{\mathrm{a}}$ & $37.5 \pm 2.5^{\mathrm{a}}$ \\
EHA105-pCAMBIA1304 & $35.0 \pm 5.0^{\mathrm{b}}$ & $87.5 \pm 7.5^{\mathrm{b}}$ \\
\hline
\end{tabular}

Values are mean $\pm S D, n=100$ with triplicated experiments. Letters indicate significant difference at $95 \%$ confidence $(p<0.05)$ via $t$-test within column.

\section{Effect of Agrobacterium strains on the transformation efficiency in dehusked seeds}

Two Agrobacterium strains (AGL1 and EHA105), carrying the binary vector pCAMBIA1304, were used to evaluate a potential of the transformation efficiency in dehusked-rice seeds. The histochemical assay of GUS transient expression in rice embryo demonstrated that the treatment of SAAT and VIAAT combination for Agrobacterium strains AGL1 $(37.5 \pm 2.5)$ and EHA105 $(87.5 \pm 7.5)$ gave significantly higher transformation efficiency than untreated AGL1 $(17.0 \pm 2)$ and EHA105 $(35.0 \pm 5)$ (used as a control). Moreover, the result revealed that the Agrobacterium strain EHA105-pCAMBIA1304 (87.5 \pm 7.5$)$ gave significantly higher transformation efficiency than AGL1pCAMBIA1304 $(37.5 \pm 2.5)$ at $p<0.05$ (Table 1). Although both strains of Agrobacterium (EHA105 and AGL1) contained supervirulent (vir) genes from oncogenic strain A281, which is able to transfer and insert a T-DNA region into various plant species, this result showed that Agrobacterium strain EHA105 was more efficient than AGL1 to transfer a T-DNA region containing gusA and nptII genes into dehuskedrice seeds. It has also been reported that Agrobacterium strain EHA105 gave higher transformation efficiency in tobacco than AGL1 [18]. Therefore, the use of this protocol should be evaluated for transformation efficiency in other rice cultivars.

\section{Effect of sonication-treatment period on microwound induction}

To investigate the influence of SAAT treatment period on microwound induction, the dehusked seeds were immersed in SIM medium and sonicated for different time durations of 5 and $10 \mathrm{~min}$ (unsonicated treatment was used as a control). All seeds were dried at $45^{\circ} \mathrm{C}$ for 2 days in an oven. The microwounds on seed surfaces were evaluated
Table 2 Transformation efficiency in different organs of germinated seeds suspended in EHA105-pCAMBIA1304 and treated with SAAT followed by VIAAT.

\begin{tabular}{lr}
\hline Plant organ & Transformation efficiency (\%) \\
\hline Coleoptile (CT) & 18 \\
Radicle (R) & 2 \\
Coleorhiza (CR) & 14 \\
Primary leaf (PL) & 2 \\
Embryo (Em) & 0 \\
Endosperm (En) & 0 \\
CT and CR & 6 \\
CT and R and PL & 4 \\
CT and CR and PL & 2 \\
CT and R and CR and PL & 0 \\
CT and R and CR & 6 \\
CT and R & 6 \\
CT and PL & 4 \\
R and PL & 2 \\
R and PL and CR & 2 \\
R and CR & 10 \\
PL and CR & 0 \\
\hline
\end{tabular}

The number of seeds is 100 seeds with 3 biological replicates in each treatment.

under photoelectron microscope (PEM). Among the different time durations, the SAAT treatment for $10 \mathrm{~min}$ was proved to be the best for increasing microwound regions on the embryo surface (Fig. 4). These microwounds may facilitate Agrobacterium to deeply penetrate into plant tissues, increasing transformation efficiency $[7,19]$.

\section{Effect of different genotypes of rice on transformation efficiency}

The transformation efficiency of the dehusked seeds of three rice cultivars (RD41, RD47 and PSL2) was examined using Agrobacterium strain EHA105pCAMBIA1301 with combination of SAAT and VIAAT techniques. The successful transformation was determined by GUS assay. The result showed that the PSL2 $(8.25 \pm 0.35 \%)$ gave significantly higher transformation efficiency in its embryo than RD41 (1\%) and RD47 (1\%) at $p<0.05$ (Duncan's new multiple range test).

\section{Influence of husked-seed germination on transformation efficiency}

To evaluate the influence of husked-seed germination on transformation efficiency, the husked seeds of rice cultivar RD41 were immersed in water for 3 days until germinated, and then dried on air for $6 \mathrm{~h}$. The germinated seeds were suspended to 
A

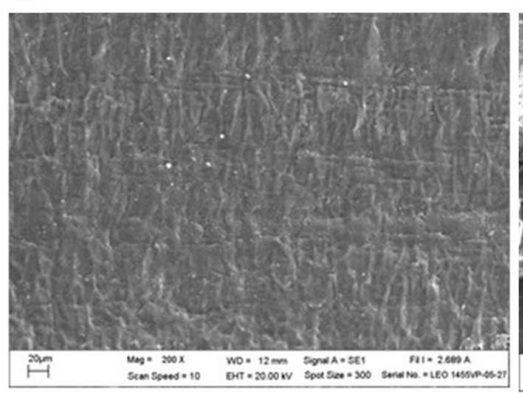

B

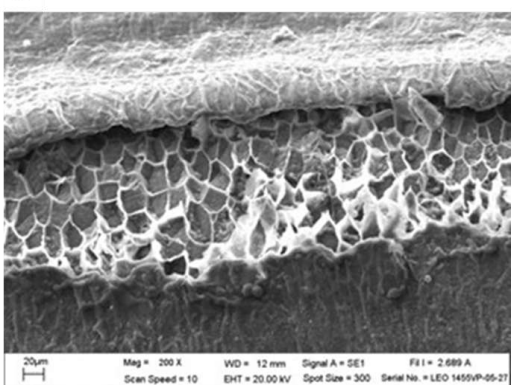

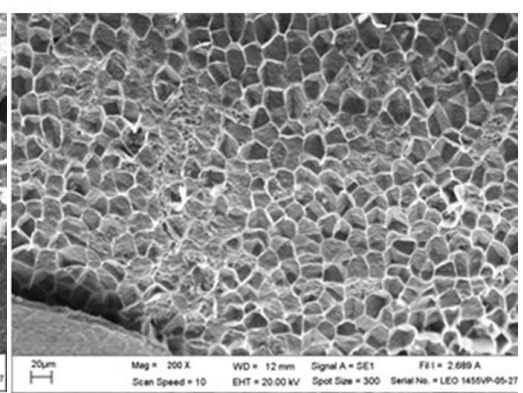

Fig. 4 Photoelectron microscope (PEM) image of microwounds on the rice-embryo surface of SAAT-treated dehusked seed (A) no sonication, (B) SAAT-treated dehusked seed for $5 \mathrm{~min}$ and (C) $10 \mathrm{~min}$. Bar indicates $20 \mu \mathrm{m}$.
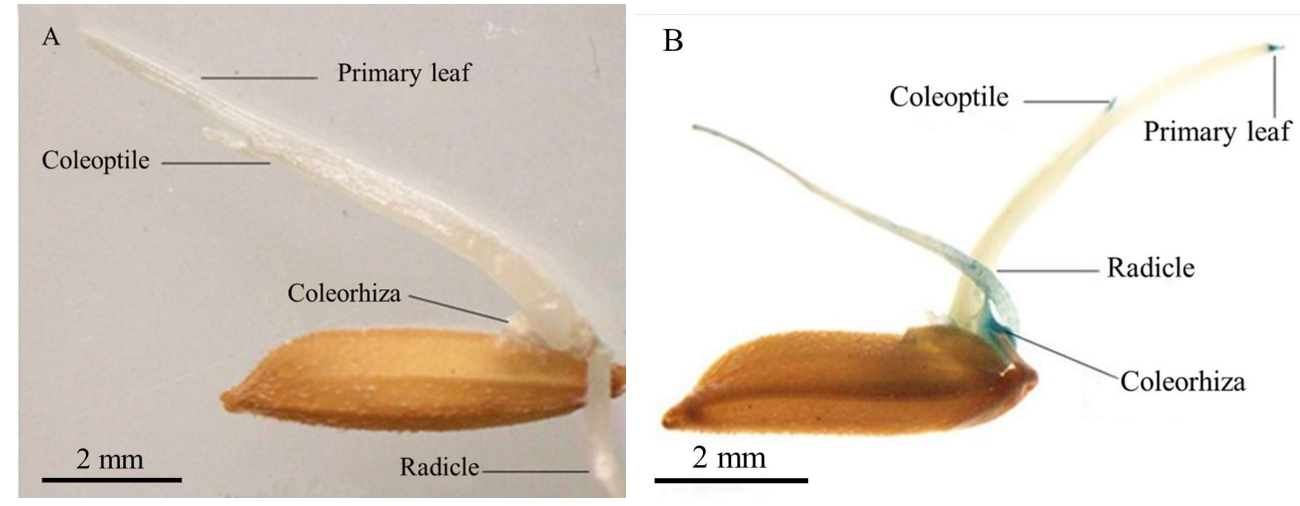

Fig. 5 Influence of husked-seed germination on transformation efficiency. Germinated seeds of RD41 rice were immersed in EHA105-pCAMBIA1304 and treated with SAAT and VIAAT followed by GUS assay in (A) control (no gusA expression) and (B) gusA expression in radicle, coleorhiza, coleoptile and primary leaf of treated germinated seeds. Bar represents $2 \mathrm{~mm}$.

EHA105-pCAMBIA1304 and treated with SAAT for $10 \mathrm{~min}$, followed by VIAAT for $10 \mathrm{~min}$. The result revealed that transient GUS expression was found in coleoptile (CT), radicle (R), coleorhiza (CR) and primary leaf (PL) of germinated seeds (Table 2, Fig. 5). The expression was high in coleoptile, coleorhiza, radicle and coleorhiza, as 18\%, 14\% and $10 \%$, respectively, whereas there was no expression of transient GUS in Em, En, CT and R and CR and PL, and PL and CR. This result indicated that the seed germination may induce wounds on surface of plant tissue that could be stimulated bacterial infection.

\section{Determination of the success of transformation by SAAT and VIAAT combination}

To determine the transient gusA expression in seedlings of rice cultivar RD41, six hundred dehusked seeds were immersed in EHA105pCAMBIA1301 and treated with SAAT for $10 \mathrm{~min}$, followed by VIAAT for $10 \mathrm{~min}$. Threehundred-dehusked seeds were determined for the transformation efficiency by GUS assay. The GUS stained areas were observed in embryo (Em) and endosperm (En) with the same level of $0.67 \%$ in one-day-old seedlings (Fig. 6A-B), and in root (Ro) and shoot (So) with $1 \%$ and $2 \%$, respectively, in two-day-old seedlings (Fig. 6C-D).

These results indicated that this protocol has the potential for the successful gene transformation by VIAAT method, especially into embryo and shoot. Embryo and shoot could be developed into germ line in both male and female that can be inherited to the next generation during the fertilization.

\section{CONCLUSION}

This work demonstrates that SAAT and VIAAT techniques could be alternatively used for direct riceembryo transformation to avoid plant tissue culture system. This will reduce the requirement of laborintensive and time-consuming process. We found that Agrobacterium strain EHA105 gave significantly higher transformation efficiency than the strain 

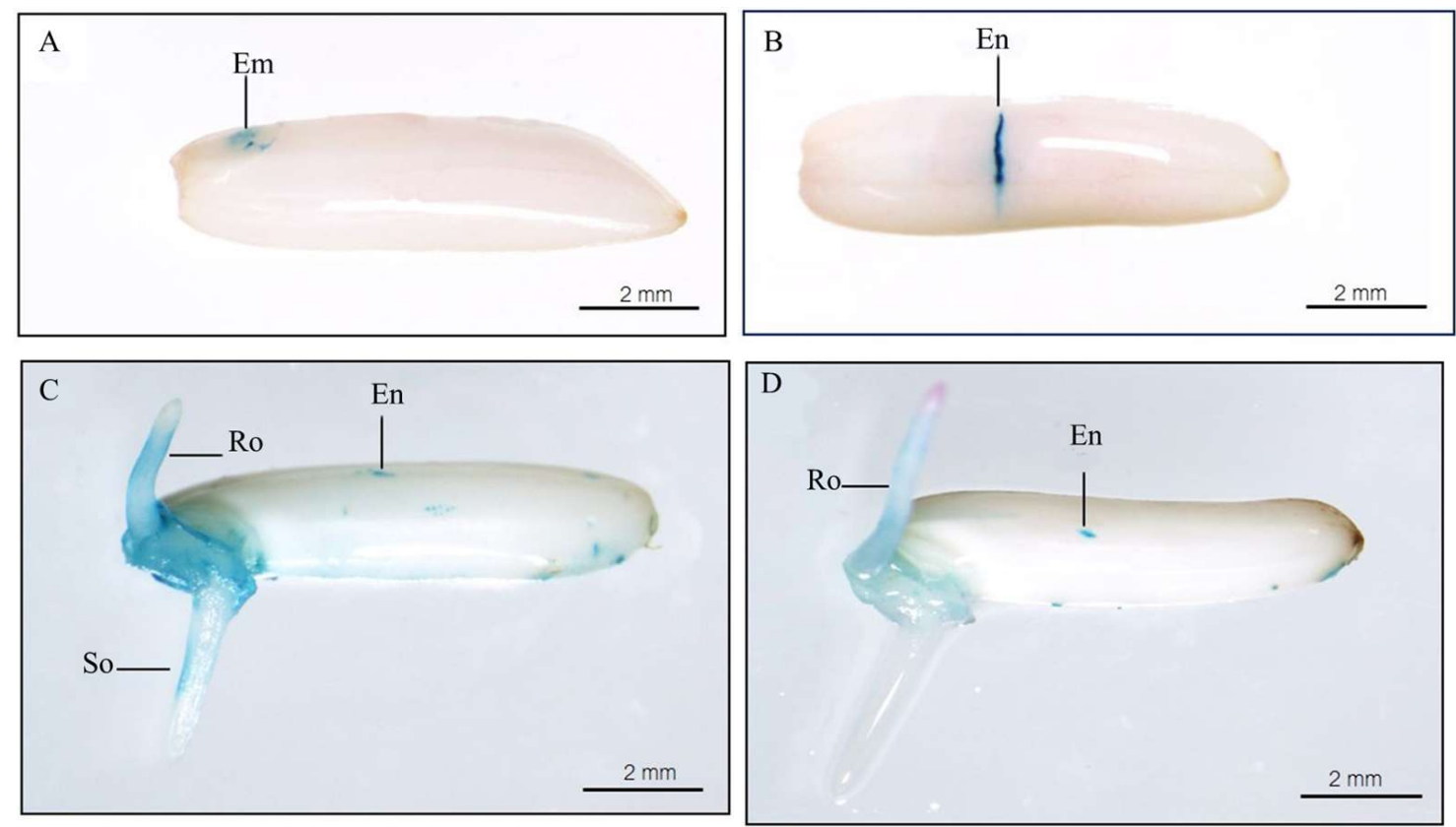

Fig. 6 Histochemical localization of gusA activity in different tissues of transformed dehusked-rice seedling at (A and B) one-day old, and (C and D) two-day old. Dehusked seeds of RD41 rice were immersed in EHA105-pCAMBIA1301 and treated with SAAT and VIAAT, followed by GUS assay. Blue-stained seedling tissues indicate gusA expression in transformed regions such as embryo (Em) (A), endosperm (En) (B, C and D), roots (Ro) (C and D) and shoot (So) (C). Bar equals to $2 \mathrm{~mm}$.

AGL1. Using SAAT for $10 \mathrm{~min}$ was proved to be the best for increasing microwound regions on the embryo surface. SAAT in combination with VIAAT led to a transfer and insert of a T-DNA region into microwound of dehusked-rice embryo induced by milling machine or sonication and into microwound of husked-rice embryo induced by seed germination. In addition, the rice cultivar PSL2 was the best for transformation efficiency when compared to RD41 and RD47.

Acknowledgements: The authors would like to thank Faculty of Agriculture, Natural Resources and Environment, Naresuan University, Faculty of Science and Technology, Pibulsongkram Rajabhat University and The Science Center, Faculty of Science and Technology, Pibulsongkram Rajabhat University. We would like to give our sincere appreciation to Prof. Dr. Sompong Te-chato from Prince of Songkla University for kindly providing A. tumefaciens strains AGL1. Thanks also go to Dr. Orawan Chatchawankanphanich from the National Center for Genetic Engineering and Biotechnology, Kasetsart University for proving A. tumefaciens strains EHA105. This research work was financially supported by a grant from National Research Council of Thailand (2558A14202028).

\section{REFERENCES}

1. Yokotani N, Sato Y, Tanabe S, Chujo T, Shimizu T, Okada K, Yamane H, Shimono M, et al (2013) WRKY76 is a rice transcriptional repressor playing opposite roles in blast disease resistance and cold stress tolerance. J Exp Bot 64, 5085-5097.

2. Hansen G, Wright M (1999) Recent advances in the transformation of plants. Trends Plant Sci 4, 226-231.

3. Hansen G, Chilton MD (2000) Lessons in gene transfer to plants by a gifted microbe. In: Hammond J, McGarvey P, Yusibov V (eds) Plant Biotechnology, Current Topics in Microbiology and Immunology, vol 240, Springer, Berlin, Heidelberg, pp 21-57.

4. Gelvin SB (2003) Agrobacterium-mediated plant transformation: the biology behind the "genejockeying" tool. Microbiol Mol Biol Rev 67, 16-37.

5. Li J, Tan X, Zhu F, Gao J (2010) A rapid and simple method for Brassica napus floral-dip transformation and selection of transgenic plantlets. Int $J$ Biol 2, 127-131.

6. Bakshi S, Sadhukhan A, Mishra S, Sahoo L (2011) Improved Agrobacterium-mediated transformation of cowpea via sonication and vacuum infiltration. Plant Cell Rep 30, 2281-2292.

7. Subramanyam K, Subramanyam K, Sailaja K, Srinivasulu M, Lakshmidevi K (2010) Highly efficient 
Agrobacterium-mediated transformation of banana cv. Rasthali (AAB) via sonication and vacuum infiltration. Plant Cell Rep 30, 425-436.

8. Chopra R, Aparna P, Saini R (2012) Use of sonication and vacuum infiltration for Agrobacterium-mediated transformation of an Indian lentil (Lens culinaris Medik.) cultivar. Sci Horticult 143, 127-134.

9. Arun M, Subramanyam K, Mariashibu TS, Theboral J, Shivanandhan G, Manickavasagam M, Ganapathi A (2015) Application of sonication in combination with vacuum infiltration enhances the Agrobacteriummediated genetic transformation in Indian soybean cultivars. Appl Biochem Biotechnol 175, 2266-2287.

10. Clough SJ, Bent AF (1998) Floral dip: a simplified method for Agrobacterium-mediated transformation of Arabidopsis thaliana. Plant $J$ 16, 735-743.

11. Insightful Science (2020) Gene mapping of pCAMBIA1301, SnapGene Software. [Available at: snapgene.com]

12. Insightful Science (2020) Gene mapping of pCAMBIA1304, SnapGene Software. [Available at: snapgene.com]

13. Thiannawa A, Kunpratum N, Ratanasut K, Sujipuli K (2015) Development of rice embryo transformation using sonication and vacuum infiltration-assisted Agrobacterium-mediated transformation. In: Proc of the 2015 Annual Conference of Maejo University, Chiang Mai, pp 126-135.
14. Jefferson RA, Kavanagh TA, Bevan MW (1987) GUS fusions: beta-glucuronidase as a sensitive and versatile gene fusion marker in higher plants. EMBO $J 6$, 3901-3907.

15. Wan Ngah WS, Hanafiah MA (2008) Removal of heavy metal from wastewater by chemically modified plant wastes as adsorbents: A review. Bioresour Technol 99, 3935-3948.

16. Beranová $M$, Rakouský $S$, Vávrová Z, Skalický $T$ (2008) Sonication assisted Agrobacterium-mediated transformation enhances the transformation efficiency in flax (Linum usitatissimum L.). Plant Cell Tiss Organ Cult 94, 253-259.

17. Zheng Q, Zheng Y, Wang G, Guo W, Zhang Z (2011) Sonication assisted Agrobacterium-mediated transformation of chalcone synthase (CHS) gene to Spring Dendrobium cultivar 'Sanya'. Afr J Biotechnol 10, 11832-11838.

18. Bakhsh A, Anayol E, Ozcan SF (2014) Comparison of transformation efficiency of five Agrobacterium tumefaciens strains in Nicotiana tabacum L. Emir $J$ Food Agric 26, 259-264.

19. Mariashibu TS, Subramanyam K, Arun M, Mayavan S, Rajesh M, Theboral J, Manickavasagam M, Ganapathi A (2013) Vacuum infiltration enhances the Agrobacterium-mediated genetic transformation in Indian soybean cultivars. Acta Physiol Plant 35, 41-54. 